\title{
Stimulus-central processing-response compatibility: Guidelines for the optimal use of speech technology
}

\author{
MICHAEL A. VIDULICH \\ NASA-Ames Research Center, Moffett Field, California \\ and \\ CHRISTOPHER D. WICKENS \\ University of Illinois, Urbana-Champaign, Champaign, Illinois
}

\begin{abstract}
With the emergence of speech technology as a viable display/control alternative, the question of guidelines is of importance. Stimulus-central processing-response (S-C-R) compatibility is proposed as a preliminary set of guidelines. S-C-R compatibility makes a two-part set of predictions about the best input/output (I/O) configuration for a task on the basis of the type of central processing that the task requires. For tasks with predominately spatial central processing demands, the best $I / O$ configuration is predicted to be visual/manual. For tasks with predominately verbal central processing demands, the best I/O configuration is expected to be auditory/speech. A series of three experiments testing these predictions is reviewed. The results are interpreted as supporting the concept of S-C-R compatibility.
\end{abstract}

Stimulus-central processing-response (S-C-R) compatibility extends the traditional concept of S-R compatibility to take into account the central processing involved in the performance of the task. S-C-R compatibility relies on a distinction between verbal and spatial processing. Depending on the primary processing demands of a task, the predicted optimal display/control configuration changes. For example, S-C-R compatibility would make the following two-part prediction regarding the use of speech technology: (1) When the primary processing demand of a task is verbal, the optimal input/output ( $\mathrm{I} / \mathrm{O})$ configuration is auditory/speech (A/S). (2) When the primary processing demand of a task is spatial, the optimal $\mathrm{I} / \mathrm{O}$ is visual/manual $(\mathrm{V} / \mathrm{M})$.

The implications of these predictions are important to system design. Given the recent development of viable computer speech synthesis and recognition equipment, it is obvious that designers of future systems will be able to make use of auditory/speech $1 / O$ in ways not previously possible. This is especially desirable in multitask environments in which heavy demands are placed almost exclusively on the visual/manual I/O channels (e.g., high-

This research was supported by the Office of Naval Research Engineering Psychology Program under Contract N000-14-79-C-0658. Gerald Malecki was technical monitor. The authors wish to thank Roger Marsh for program development in Experiments 1 and 3. For Experiment 2, we wish to express our appreciation to Sam Schiflett, for help in developing the experiment and acquiring access to the equipment, and to Joe Alfano for program development. Christopher D. Wickens is with the Institute of Aviation and Department of Psychology, University of Illinois, Champaign, IL 61820. Requests for reprints should be sent to Michael Vidulich, Mail Stop 239-3, NASA-Ames Research Center, Moffett Field, CA 94035. performance aircraft, some process control tasks). However, since computer speech technology is of such recent vintage, there is no proven set of guidelines to control its application. In this paper, we will attempt to show that the concept of S-C-R compatibility is a useful guideline for the application of speech technology.

The organization of the paper is as follows: (1) A review of the logic underlying the formulation of S-C-R compatibility is undertaken. This begins with a brief review of stimulus-response (S-R) compatibility, the logical precursor to S-C-R compatibility. (2) The assumed distinction between verbal and spatial central processing codes and the predictions of the S-C-R compatibility principle are described. (3) The results of a research program to evaluate its viability are discussed. And, (4) a brief recapitulation and a discussion of implications are presented.

\section{THE LOGIC OF S-C-R COMPATIBILITY}

S-R compatibility was originally defined by Fitts and Seeger (1953) as follows:

A task involves compatible S-R relations to the extent that the ensemble of stimulus and response combinations comprising the task results in a high rate of information transfer .... This interpretation makes use of the idea of a hypothetical process of information transformation or recoding in the course of a perceptualmotor activity, and assumes that the degree of compatibility is at a maximum when recoding processes are at a minimum. (p. 199, italics added) 
Although Fitts and Seeger (1953) mentioned that S-R compatibility can be extended to "simultaneous listening and looking" (p. 199), they chose to focus on the issue of compatibility of the spatial characteristics of a stimulus array and its associated responses. Fitts and Seeger showed that increasing compatibility is advantageous to performance, and that the advantage persists even after extensive practice. In the years since this classic paper, the concept of S-R compatibility has, for the most part, come to be limited to this specific domain: namely, an agreement in directions of movement between displays (i.e., stimuli) and controls (i.e., responses). As such, this conception of compatibility does not readily lend itself to guiding the application of speech technology.

S-C-R compatibility attempts to rectify this by extending compatibility to include consideration of the type of central processing required by performing a given task. The assumption is made that there are two main types of information processing possible from a human operator: verbal processing and spatial processing. Verbal processing is used in dealing with semantic information and is most evident in tasks requiring logical linguistic operations (e.g., radio communications and mental arithmetic). Spatial processing encompasses information and operations that are analog in nature and require mental transformations along the six axes of translation and rotation (e.g., navigation, tracking, target localization). This dichotomization of processing types is common to numerous theories and has garnered much empirical support (see Gazzaniga, 1970, Kinsbourne \& Hicks, 1978, Leiber, 1982, Pavio, 1971, Posner, 1978, and Wickens, 1980). Although it is certainly true that numerous real-world tasks are complex enough to involve a combination of both types of processing, the assumption is made here that many tasks can be usefully classified as one or the other.

Given that the distinction can be made between the two types of processing, why would one expect that the optimal I/O channel for a task would be influenced by the type of processing that the task employs? Over the years, a variety of experimental results, considered collectively, suggest that spatial tasks are best served by visual/manual $\mathrm{I} / \mathrm{O}$ and verbal tasks by auditory/speech $\mathrm{I} / \mathrm{O}$. Since this literature has already been reviewed in a previous paper (i.e., Wickens, Sandry, \& Vidulich, 1963), it will not be reviewed here.

However, it is important to carefully review the testable predictions of S-C-R compatibility. These predictions are presented graphically in Figure 1. Figure 1a shows the expected single-task performance in both a spatial task and a verbal task as the $\mathrm{I} / \mathrm{O}$ configuration is changed from auditory/speech to visual/manual. In both cases, the prediction is that performance will be optimal when the S-C-R compatible configuration is employed, reduced when either the input or the output is incompatible, and at its worst when both input and output do not conform to S-C-R compatibility. Figure $1 \mathrm{~b}$ shows how performance would be predicted to fare if these single-task conditions were performed concurrently with a $\mathrm{V} / \mathrm{M}$ spatial
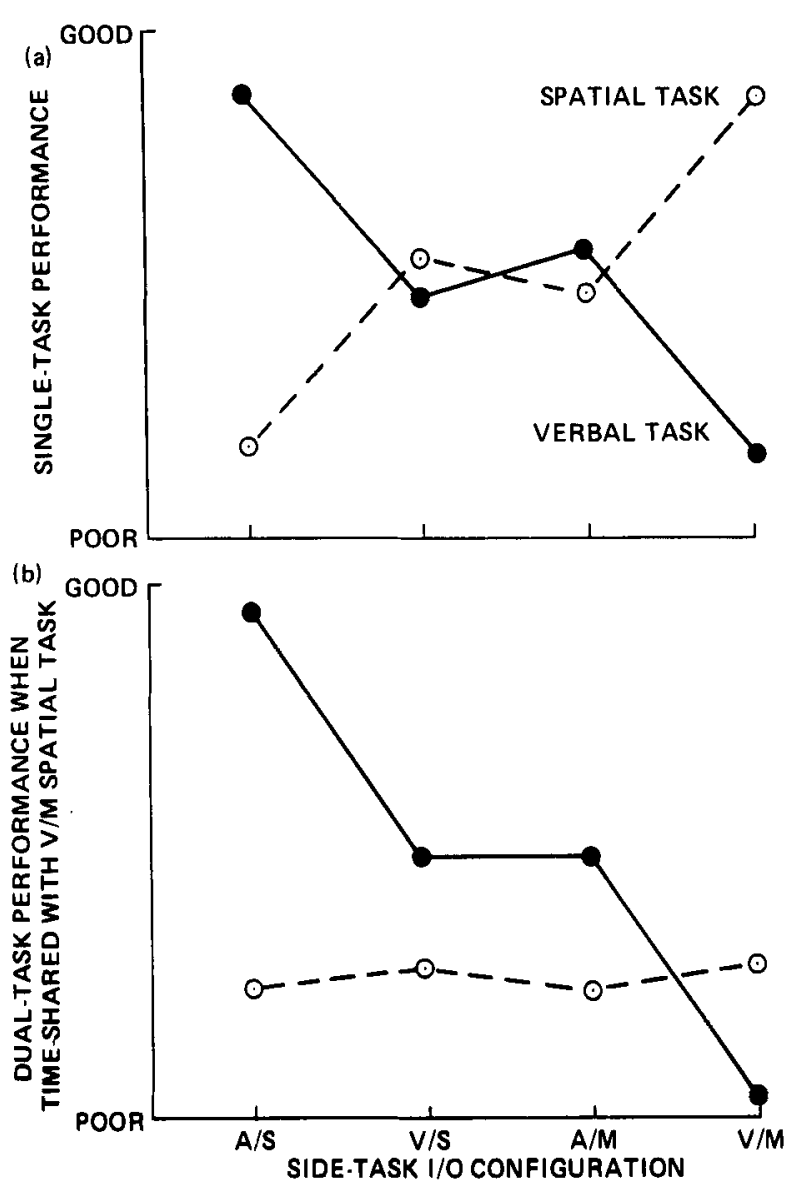

Figure 1. Theoretical predictions of performance on spatial and verbal tasks as the input/output (1/O) configuration is changed from auditory/speech (A/S) to visual/manual (V/M): (a) single-task performance, (b) time-sharing performance when time-shared with a primary $\mathrm{V} / \mathrm{M}$ spatial task.

task (such as flying an aircraft). Here, the advantages of S-C-R compatibility are expected to be attenuated in the original spatial task because they are pitted against increasing competition for the $1 / 0$ channels, and therefore the curve for spatial task performance is flattened out at a somewhat poor level. For the verbal task, however, the expected effects of S-C-R compatibility and I/O competition are in complete agreement. Consequently, the prediction is that the effects of changing $\mathrm{I} / \mathrm{O}$ configuration will appear to be enhanced compared with the singletask effects.

These predictions were tested in a series of three experiments. The results of these experiments will be discussed next.

\section{S-C-R COMPATIBILITY EXPERIMENTS}

\section{Experiment 1: Time-Sharing Tracking and Memory Search}

Ten right-handed male subjects time-shared a onedimensional compensatory tracking task with a memory search task. The tracking task was always performed in a visual/manual $1 / O$ configuration and is considered to be 
a spatial task. The tracking task's difficulty was manipulated by changing the control dynamics from first-order velocity control to second-order acceleration control. The memory search task required subjects to remember three randomly selected letters. During a trial, probe letters were presented periodically and the subjects responded as quickly as possible, indicating whether the probe letter was a memory set item or not. The letters were presented either visually on the same CRT screen that displayed the tracking task or auditorily through the subjects' headphones. Subjects' responses were collected either by buttonpresses or by a speech recognition unit. The memory search task was performed in all four possible I/O configurations (i.e.,, auditory/speech, auditory/ manual, visual/speech, and visual/manual), both alone and paired with the tracking task. In short, this first experiment was a laboratory test of the verbal condition predictions represented by the solid lines and circles on Figure 1 . A more detailed review of this first experiment is available in Vidulich and Wickens (1981).

Results. The results are graphically displayed in Figure 2. In Figure 2a, the single-task accuracy for the memory search task is displayed. Note that the percent error scale has been inverted so that "good" performance is toward the top of the figure. An ANOVA performed on these data indicated that: Auditory input was superior to visual input $[F(1,9)=8.6, p<.02]$, speech response was superior to manual response $[F(1,9)=7.8, p<.03]$, and input modality interacted with output modality $[\mathrm{F}(1,9)$ $=38.4, \mathrm{p}<.01]$. The interaction appears to result from an enhancement of the speech output superiority in the visual input condition.

The single-task memory search latency data were analyzed with the same statistical procedure as the error data. It was found that: Visual responses were faster than speech responses $[F(1,9)=77.0, p<.01]$, manual responses were faster than speech responses $[F(1,9)=355.0, p<$ $.01]$, and there was no significant interaction. These results contradict the predictions of S-C-R compatibility, but are likely to be the result of peripheral timing effects. For the input effect, timing for both the visual input and the auditory input began with the onset of the stimulus. Consequently, the visual display contained all relevant information at the moment the timing began, whereas the auditory format inherently required time to present its information. On the response side, the latency data include the computer's response recognition time, which was substantially shorter in the manual output configurations. Because of these timing confounds, the single-task latency data are considered unimportant in evaluating the claims of S-C-R compatibility, which are concerned primarily with the efficiency of central processing. In the singletask case, error frequency seemed to be the better indicator of central processing efficiency.

However, in the dual-task case, the latencies can be used effectively by the simple expedient of generating decrement scores. Decrement scores are calculated by subtracting the appropriate single-task latency from each dual-
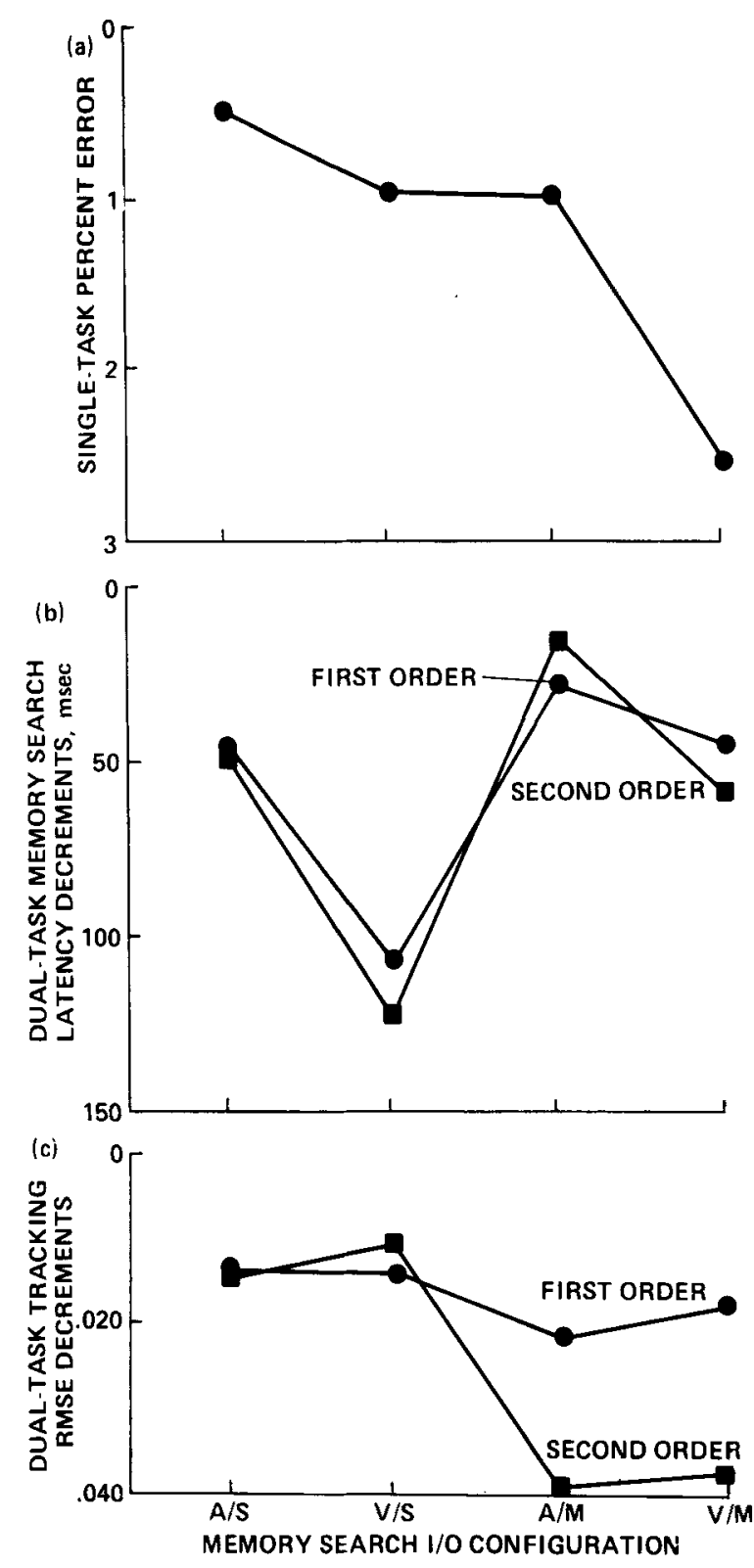

Figure 2. Performance in Experiment 1.

task latency. This difference is an estimate of the effect on processing efficiency of having to perform the memory search and tracking tasks concurrently with the singletask timing contaminations removed. These data are displayed in Figure $2 b$. The curve with the circles shows the memory search latencies when performed with a concurrent first-order tracking task. The curve marked with the squares shows the corresponding memory search latencies when the concurrent tracking task is second order. The two curves lie close together, reflecting that the control order of the concurrent tracking task had no effect on memory search latency decrements $(p>.6)$. Inconsistent with the predictions, the manual output conditions resulted in a smaller decrement than the speech output conditions $[F(1,9)=9.3, p<.02]$. However, the audi- 
tory input was superior to the visual input $[F(1,9)=8.9$, $\mathrm{p}<.02$ ], as predicted by S-C-R compatibility. No significant interaction between input and output modalities was detected $(\mathrm{p}>.1)$.

The inconsistent effect of output modality on memory search latencies is clarified when the decrement scores of the concurrent tracking task are examined. Remember that the predictions in Figure $1 \mathrm{~b}$ are for dual-task performance; therefore, the performance in both tasks must be considered. The tracking data, displayed in Figure 2c, show a strong interaction between the control order of the tracking task and the output modality of the memory search task $[F(1,9)=22.2, p<.01]$. When the tracking task is second order and the memory search output is manual, the decrements are large. When either of these conditions is not met, the decrements are small.

Conclusions. The results of this experiment can be interpreted as substantially supporting the predictions of S$\mathrm{C}-\mathrm{R}$ compatibility. The single-task memory search error data clearly show the predicted pattern. The results of the dual-task analyses considered as a whole are also encouraging. When the memory search task has either a visual input or a manual output, some aspect of the dualtask performance suffers. The memory search task, a cognitive task, is disrupted by common inputs, whereas tracking, a heavily response-oriented task, is disrupted by common outputs. This finding is consistent with the predictions of multiple resource theory (Wickens, 1980).

Therefore, the results of the first study are interpreted as support for the concept of S-C-R compatibility as applied to the application of speech technology. However, two deficiencies need to be noted: First, only half of the predictions of S-C-R compatibility were tested. The question of a spatial task time-shared with the tracking task was not addressed at all. Second, the S-C-R compatibility manipulation was confounded with competition for the same I/O channels. As S-C-R compatibility was decreasing, I/O competition was increasing. Further research is needed to examine these problems.

\section{Experiment 2: S-C-R Compatibility in an F-18 Simulator}

The second experiment expanded on the first in two ways: (1) A spatial target-localization side task and a verbal communications side task were employed to give a more thorough test of the predictions of S-C-R compatibility. (2) The primary V/M spatial task was to fly a mock-up F-18 simulator through a course shown on a "tunnel-in-the-sky" display. This represents a considerable increase in fidelity over the compensatory tracking task used in the first experiment.

Ten right-handed male general aviation pilots served as the subjects in this experiment. The primary flight task was always presented visually on a simulated heads-up display (HUD) and was responded to manually. The task required subjects to fly through a three-dimensional tunnel displayed on the HUD. Difficulty of this task was manipulated by varying the speed that subjects were re- quired to maintain. In the easy condition, subjects were required to maintain $300 \mathrm{knots}$, and in the hard condition, 500 knots. Performance was summarized in a figureof-merit (FOM) score, a weighted index that accounted for horizontal, vertical, and air speed error. This task was considered to be a spatial task.

One side task was a verbal communications task. In this task, commands such as "turn on radar-beacon, tacan" or "enter latitude north 2142 " were presented to the subjects either visually on the HUD display or auditorily. The subjects were required to respond either manually with the free hand on a keyboard or vocally into a speech recognition unit. Hence, all four I/O configurations were employed.

The second side task was a spatial target localization task. Potential targets (e.g., factories, houses, hangers) were drawn on the "ground" of the HUD display. Periodically, a class of target was specified either by writing its name at the bottom of the HUD display or by speaking the name auditorily. The subjects' task was to position a cursor over the specified target. The cursor could be moved manually or by vocal commands. As in the verbal side task, the spatial side task was performed in all four I/O combinations.

The combination of two levels of the primary flight task and eight possible side tasks resulted in a total of 26 experimental conditions (10 single-task, 16 dual-task). After reaching a criterion level of performance, each subject performed all 26 conditions six times each. For more detail about this experiment, refer to Sandry and Wickens (1982).

Results. The data, collapsed across replications and primary task difficulty levels, are displayed in Figure 3. In Figure 3a, the single-task performance for the two side tasks is displayed. Unlike in the first experiment, the latency data are presented. In this experiment, to adjust for inherent differences in stimulus types, timing began at the end of the auditory message. This could lead to an underestimate of processing time if the subjects began processing before the end of the signal. Error rates ranged from $2 \%$ to $4 \%$ with the lowest error rates associated with the fastest latencies.

Figure 3a shows a strong resemblance to the predictions made in Figure 1. Side task type interacted with both input modality $[\mathrm{F}(1,7)=89.43, \mathrm{p}<.0001]$ and output modality $[F(1,7)=48.28, p<.0001]$. Response latencies for the auditory input were faster than those for the visual input in the verbal side task, but this trend was reversed in the spatial side task. The speech output resulted in faster latencies than the manual output in the verbal task and slower latencies in the spatial task. All in all, the data were in complete agreement with the predictions.

Figure $3 b$ shows the latency decrements for the two side tasks when performed concurrently with the primary flight task. As predicted, there was a significant input modality $\times$ task interaction $[F(1,7)=41.75, p<.001]$. The timesharing efficiency of the verbal side task was helped by 

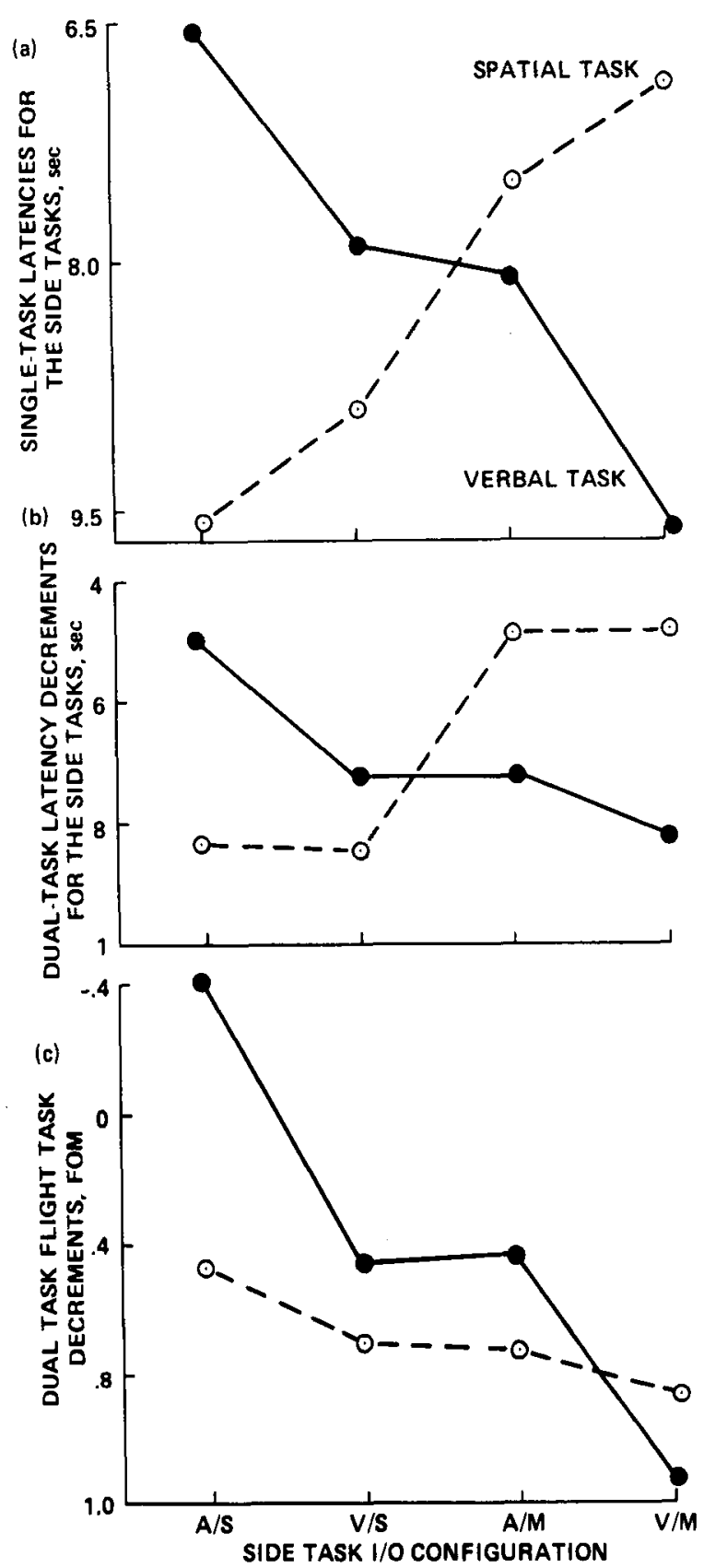

Figure 3. Performance in Experiment 2.

auditory input, whereas the efficiency of the spatial task was not. However, the significant interaction between output modality and task $[\mathrm{F}(1,7)=39.74, \mathrm{p}<.001]$ was not as anticipated. The verbal task showed the expected trend, but the manual responses had an unexpected advantage for the spatial task. Apparently, the advantage of the S-C-R compatible output overwhelmed the increasing competition for the manual response resources. One other aspect of the predictions of Figure $1 \mathrm{~b}$ failed to emerge in these data: The spatial task was expected to suffer greater interference than the verbal task as a result of central processing resource competition. However, no main effect of task was detected $(p>.1)$.
On the other hand, the spatial processing interference effect that failed to show in the side task data was evident in the primary flight task data. These data are displayed in Figure 3c. In this figure, which is an almost perfect duplicate of Figure 1b, the significant effect of task type is clear $[F(1,7)=5.75, p<.05]$. The spatial side task interfered with the primary flight task to a greater degree than did the verbal task.

Conclusions. Experiment 2 replicated and extended the resuits of Experiment 1. The verbal side task produced effects consistent with both the predictions and the findings of Experiment 1. The spatial side task displayed the expected effects as a single task, but, when time-shared with the flight task, showed an unexpectedly robust effect of output compatibility despite competition for output resources. In effect, S-C-R compatibility was even stronger than predicted. Given that these findings were obtained in an environment with strong fidelity to realworld tasks, they argue strongly for the value of S-C-R compatibility as a design guideline.

However, one weakness of Experiment 1 persisted through Experiment 2 . Since the primary flight task is always a spatial task, the S-C-R compatibility manipulation was still confounded with resource competition. In the verbal task, resource competition aided the expected effects of S-C-R compatibility, whereas in the spatial task, it opposed them. The third experiment was designed to correct this deficiency.

\section{Experiment 3: S-C-R Compatibility Unconfounded by Resource Competition}

In the third experiment, it was decided to create two tasks, one verbal and one spatial, both of which could be varied across the four $\mathrm{I} / \mathrm{O}$ configurations. These two tasks could then be used to examine the effects of S-C-R compatibility independently of resource competition. The two tasks were called the "fault" task and the "threat" task. The fault task was an abstract troubleshooting task that required the subjects to work through a hierarchical checklist of systems and parts to identify those that had failed and remember them until the appropriate time to report. The subject interrogated the computer either vocally or via a special keyboard and the computer responded either auditorily or via a CRT display. The second task, the threat task, required subjects to evaluate the threat of collision with another aircraft on the basis of that aircraft's position and relative heading. Information about the other aircraft could be presented on the CRT or via a pair of tones. Subjects' responses were collected from either a second special keyboard or by speech recognition. Obviously, the threat task is a spatial task, whereas the fault task is verbal.

Since each task had four possible I/O configurations, it logically follows that there were 16 possible dual-task pairs. However, 4 of these 16 possible conditions would have required subjects to speak two things simultaneously and were therefore deleted from the set. Twenty subjects were run in this experiment.

Results. Because of the large number of data gener- 
ated in this experiment and the complexity of the design, it is impossible to present a detailed description of the results here. These data were presented in detail in Wickens and Vidulich (1982). In the present paper, only some representative trends will be discussed.

One analysis focused specifically on S-C-R compatibility under conditions of equivalent resource competition. In this analysis, 2 of the 12 dual-task conditions were selected for direct comparison. One condition was the A/S fault task paired with the V/M threat task, the most S-C$\mathrm{R}$ compatible configuration possible. This was compared with the least compatible configuration, the $\mathrm{V} / \mathrm{M}$ fault task paired with $\mathrm{A} / \mathrm{S}$ threat task. In this analysis, $\mathrm{I} / \mathrm{O}$ resource competition did not change; neither combination inflicted any $\mathrm{I} / \mathrm{O}$ resource competition. Consequently, this analysis can be considered a pure test of S-C-R compatibility. The analysis revealed that the difference between these conditions was consistent and large. When the I/O configurations were made incompatible, the threat task's latency and accuracy decrements were increased $[F(1,9)$ $=6.64, \mathrm{p}<.03$, and $\mathrm{F}(1,9)=17.40, \mathrm{p}<.01$, respectively]. The time-per-operation decrement in the fault task increased in the incompatible condition $[\mathrm{F}(1,9)=11.00$, $\mathrm{p}<.01]$. The fault task accuracy declined, but not significantly.

Another example, exemplifying the types of effects obtained in this experiment, is displayed in Figure 4. This figure shows the dual-task latency decrements of the threat task as a function of the $\mathrm{I} / \mathrm{O}$ configuration of the fault task. The compatibility of the $\mathrm{I} / \mathrm{O}$ configuration of the fault task decreases from left to right. The data for the $\mathrm{V} / \mathrm{M}$ and $\mathrm{A} / \mathrm{M}$ threat tasks are plotted. The time-sharing efficiency declined as the fault task's S-C-R compatibility was

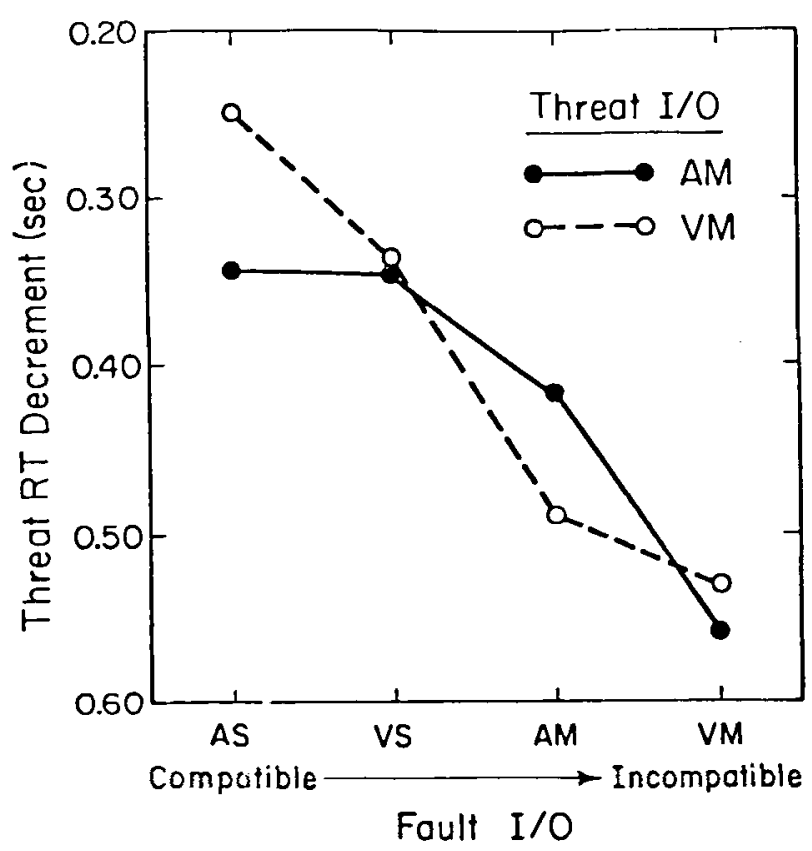

Figure 4. Effects of fault task compatibility on threat task latency decrements in Experiment 3. reduced. This is particularly evident with the visual threat display, represented by the dashed line, in which incompatibility and resource overlap are perfectly correlated. The threat task reaction time decrements showed a reliable main effect of fault input $[F(1,18)=5.99, p<.03]$ and a three-way interaction of threat input with fault input and fault output $[F(1,18)=4.85, p<.05]$. This interaction appears to result from the large decrement of the A/M threat task when time-shared with the A/S fault task. This might have been caused by an overload of the auditory channel. In this particular combination of tasks, the subjects had to attend to three sources of auditory information: stimuli for the two tasks and feedback of their vocal responses in the fault task.

Conclusions. The results of Experiment 3 show that the effects of S-C-R compatibility still occur when the compatibility is varied independently of resource competition. This lends considerable support to the previous conclusions of the first two experiments.

\section{GENERAL DISCUSSION}

On the basis of the research reported in this paper, three general conclusions relevant to the use of S-C-R compatibility in man-machine interfaces can be made.

Considerable gain can be achieved by employing the auditory/speech mode of display/control interactions in cognitive side tasks. In all three experiments, dual-task conditions were performed better when the $\mathrm{I} / \mathrm{O}$ operations were divided across both visual/manual and auditory/speech than when only visual/manual channels were used.

The assignment of tasks to the auditory/speech $1 / 0$ alternative should not be done arbitrarily. The research clearly shows that the value of speech technology interfaces varies considerably with the type of central processing demanded by the task involved.

When possible, the greatest gain from speech technology will be achieved when it is used as the interface for a task that requires primarily verbal central processing. Similarly, tasks involving primarily spatial central processing are performed better with a visual/manual interface. The experimental findings show that both singletask performance and dual-task time-sharing efficiency are at their maximum when I/O configuration is consistent with the prescription of S-C-R compatibility. In some cases, for example the manual response conditions of the spatial side task in Experiment 2, the S-C-R compatibility effect overwhelmed the expected attenuation of performance from competition for $\mathrm{I} / \mathrm{O}$ channels.

Nothing about these findings appears to be counterintuitive, but it is hoped that this research will help promote awareness of the relationship between speech technology and compatible display/control design. The value of S-C-R compatibility as a design guideline would seem to be particularly high in those operational environments that couple a demanding spatial primary task with visual/manual $\mathrm{I} / \mathrm{O}$, especially if that task inflicts high 
workload. Recall that increasing the control order of the tracking task enhanced the value of compatible I/O configurations in the first experiment. Many flight tasks would certainly appear to meet this criterion.

On a theoretical level, S-C-R compatibility is an extension of the basic conception put forward by Fitts and Seeger (1953). As pointed out in the quotation cited earlier, the concept of compatibility is based on the idea that, by requiring a minimum of recoding or information transformations, the cognitive processing related to a task can be streamlined and maximum performance achieved. The traditional S-R compatibility concept was applied strictly to the design of spatial array of the display/control interface. S-C-R compatibility adds consideration of the type of primary central processing required by task performance. If the $\mathrm{I} / \mathrm{O}$ modality and format are made compatible with the type of processing required by a task, the number of recodings, or transformations, that must be performed will diminish. It is hoped that S-C-R compatibility, by taking type of central processing into account, has increased the applicability of compatibility as a design tool without harming the simple elegance of the original S-R compatibility concept.

\section{REFERENCES}

FrTtS, P. M., \& SEEger, C. M. (1953). S-R compatibility: Spatial charac- teristics of stimulus and response codes. Journal of Experimental Psychology, 46, 199-210.

Gazzaniga, M. S. (1970). The bisected brain. New York: Meredith. KinsBourne, M., \& Hicks, R. E. (1978). Functional cerebral space: A model for overflow, transfer and interference effects in human performance: A tutorial review. In J. Requin (Ed.), Attention and performance VII (pp. 345-362). Hillsdale, NJ: Erlbaum.

LEIBER, L. (1982). Interhemispheric relationships in cognitive processing. In R. N. Malatesha \& L. C. Hartlage (Eds.), Neuropsychology and cognition (Vol. 2, pp. 235-251). The Hague: Martinus Nijhoff.

PAvio, A. (1971). Imagery and verbal processes. New York: Holt, Rinehart \& Winston.

PosNer, M. I. (1978). Chronometric explorations of mind. Hillsdale, NJ: Erlbaum.

SANDRY, D. L., \& WICKENS, C. D. (1982). The effect of stimulus-central processing-response compatibility and resource competition on pilot performance (Tech. Rep. No. EPL-82-1/ONR-82-1). Champaign: University of Illinois, Engineering-Psychology Research Laboratory.

VIDULICH, M., \& WiCkENS, C. D. (1981). Time-sharing manual control and memory search: The joint effects of input and output modality competition, priorities, and control order (Tech. Rep. No. EPL81-4/ONR-81-4). Champaign: University of Illinois, EngineeringPsychology Research Laboratory.

WiCKENS, C. D. (1980). The structure of attentional resources. In R. S. Nickerson (Ed.), Attention and performance VIII (pp. 239-257). Hillsdale, NJ: Erlbaum.

Wickens, C. D., SANDry, D., \& Vidulich, M. (1983). Compatibility and resource competition between modalities of input, central processing, and output. Human Factors, 25, 227-248.

WickENS, C. D., \& VIDULICH, M. (1982). S-C-R compatibility and dual task performance in two complex information processing tasks: Threat evaluation and fault diagnosis (Tech. Rep. No. EPL-82-3/ONR-823). Champaign: University of Illinois, Engineering-Psychology Research Laboratory. 